\title{
LncRNA BANCR Attenuates the Killing Capacity of Cisplatin on Gastric Cancer Cell Through the ERKI/2 Pathway
}

This article was published in the following Dove Press journal: Cancer Management and Research

\author{
Xiang Miao (D) \\ Yixiang Liu iD \\ Yuzhu Fan iD \\ Guoqiang Wang iD \\ Hongbo Zhu iD \\ Department of General Surgery, \\ Lianyungang Municipal Oriental Hospital, \\ Lianyungang, Jiangsu 222042, People's \\ Republic of China
}

Correspondence: Hongbo Zhu Department of General Surgery, Lianyungang Municipal Oriental Hospital, No. 57, Zhonghua West Road, Lianyungang, Jiangsu 222042, People's Republic of China

$\mathrm{Tel}+86-518-80683108$

Fax +86-5I8-8068I08

Email hnmyh1993@I63.com
Purpose: Chemotherapy-based comprehensive treatments are the most important therapeutic methods for patients with advanced gastric cancer, but chemoresistance often cause treatment failure. Long non-coding RNA (LncRNA) BRAF-activated non-coding RNA (BANCR) has been shown to participate in many biological behaviors of multiple cancers. However, the biological roles of LncRNA BANCR in chemoresistance of gastric cancer remain unclear. Here, we aimed to evaluate the functions of LncRNA BANCR on the therapy of gastric cancer.

Methods: In this study, LncRNA BANCR expression was detected in gastric cancer patient samples and cell lines by quantity polymerase chain reaction (qPCR). Cell proliferation and viability in cisplatin-treated cells were measured using clonogenic survival assay and cell counting kit-8. The levels of ERK1/2 pathway molecules were tested with Western blot. Ly3214996, an inhibitor of ERK signal pathway, was administered to assess the effects of BANCR overexpression on gastric cancer cell with cisplatin-treated resistance. Moreover, the role of BANCR in cisplatin resistance of gastric cancer was validated in xenograft mouse models in vivo.

Results: Our study revealed that LncRNA BANCR expression was also significantly increased in gastric cancer tissues compared with adjacent normal tissues. Furthermore, we found that BANCR overexpression promoted gastric cancer cell resistance to cisplatin in vitro. Ly3214996 treatment abolished the BANCR overexpression-mediated gastric cancer cell cisplatin resistance via regulating the phosphorylation of ERK protein. Knock-down of BANCR significantly delayed tumor growth in xenograft mouse models.

Conclusion: BANCR promoted cisplatin resistance of gastric cancer cells by activating ERK1/2 pathway. Inhibition of BANCR markedly suppressed the growth of gastric cancer cells in vitro as well as in vivo. These results provided a new strategy for gastric cancer therapy via targeting BANCR.

Keywords: BANCR, chemoresistance, cisplatin, ERK1/2 pathway, gastric cancer

\section{Introduction}

Gastric cancer (GC) is the fourth most common malignant tumor and the second leading cause of cancer-correlated death all over the world. ${ }^{1,2}$ Surgery is currently the most effective treatment for non-metastatic GC. ${ }^{3}$ However, more than three quarters of patients with GC were diagnosed at advanced stages and metastasis, where tumors could not be surgically removed. Therefore, chemotherapy-based comprehensive treatments are the most important therapeutic tools for patients 
with advanced GC. However, treatments have failed in many patients due to chemoresistance, which are the biggest obstacles to overcome for the successful cure of GC, and solving this problem is an urgent concern.

Emerging evidence suggested that long non-coding RNAs (LncRNAs) controls tumorigenesis and development through regulating expressions of target genes. ${ }^{4-6}$ Importantly, a number of aberrant LncRNAs have been reported to be involved in cancer chemoresistance. ${ }^{7,8}$ For example, LncRNA MALAT1 silenced sensitive GC cells to autophagy were associated chemotherapeutics via competing endogenous RNA for miR-23b-3p. ${ }^{2}$ Unregulated LncARSR promoted doxorubicin resistance via regulating PTEN-PI3K/Akt pathway in hepatocellular carcinoma. ${ }^{9}$ LncRNA BLACAT1 accelerated the oxaliplatin-resistance through upregulating ABCB1 expression by targeting miR361 in GC. ${ }^{10}$ These data indicated that LncRNAs play a critical role in the chemoresistance of various cancers.

BRAF-activated non-coding RNA (BANCR), first identified by Flockhart et al ${ }^{11}$ exerts crucial roles in regulating cell proliferation and migration in malignant melanoma. $\mathrm{Li}$ et $\mathrm{al}^{12}$ reported that the level of BANCR was upregulated in GC tissues and was positively associated with clinical stage, tumor depth, lymph node metastasis (TNM), and distant metastasis in GC patients. Furthermore, high expression of BANCR was a poor independent prognostic factor for GC patients. ${ }^{12}$ However, the biological roles of BANCR in chemoresistance of GC remain unclear. In this study, LncRNA BANCR expression level was investigated in $\mathrm{GC}$ tissues and adjacent normal tissues. We also explored the mechanism of LncRNA BANCR in cisplatin-based chemoresistance in GC patients.

\section{Materials and Methods}

\section{Cell Lines, Clinical Samples, Animals, and Reagents}

The GES-1 is a non-malignant gastric epithelial cell line. AGS, HGC27, SGC7901, and MKN45 are gastric cancer cell lines with different differentiation degrees. These cell lines were obtained from Shanghai Institute of Biochemistry and Cell Biology, Chinese Academy of Sciences (Shanghai, China) and were cultured at $37^{\circ} \mathrm{C}$ in $5 \% \mathrm{CO}_{2}$. AGS: human gastric adenocarcinoma cell; HGC 27, human gastric cancer cell with metastatic lymph node; SGC7901: human gastric cancer cell (lymph node metastasis of patients with gastric adenocarcinoma); MKN45: human gastric cancer cell. The GES-1 and SGC7901 cells were cultured in Roswell Park
Memorial Institute-1640 (RPMI)-1640 (Gibco, Rockville, MD, USA), while AGS, HGC27, and MKN45 cells were cultured in Dulbecco's modified Eagle's medium (Gibco). All media were supplemented with $10 \%$ fetal bovine serum (Hyclone, USA), 100 unit $/ \mathrm{mL}$ penicillin, and $100 \mathrm{mg} / \mathrm{mL}$ streptomycin (100 mg/mL) (Invitrogen, Carlsbad, CA, USA).

Thirty-five paired cancer and adjacent normal tissues from patients with gastric cancer who underwent surgical resection were included in this study. In the 35 GC cases, there were 20 male and 15 female patients with a median age of 64.2 year-old (range 23-87 year-old). This study protocol was approved by the ethics committee of the Lianyungang Municipal Oriental Hospital (Lianyungang, Jiangsu, China). In addition, we obtained informed consent from each patient before the study.

Six to eight-week-old female BALB/C nude mice were purchased from the experimental animal center of Shanghai, China and fed in a pathogen-free mice facility, which were cultured at $22-25^{\circ} \mathrm{C}$ temperature and $40-60 \%$ humidity, respectively. All animal study protocol was approved by our hospital ethical committees.

Cisplatin and ERK inhibitor Ly3214996 were purchased from Sigma (Sigma, USA, Cat\#: BP809 for cisplatin and A668057 for Ly3214996) and stored at 10mmol/L stock in dimethyl sulfoxide (DMSO) solution.

\section{Cell Transfection and Infection}

Expression plasmids encoding BANCR and corresponding empty vectors as control were obtained from GenePharma Co. Ltd. (Shanghai, China). Three BANCR siRNAs were also obtained from GenePharma Co. Ltd. GC cells were transfected with BANCR plasmids or BANCR siRNAs using Lipofectamine 2000 (Invitrogen, USA) according to the manufacturer's instructions.

\section{RNA Isolation and qRT-PCR}

Total RNA was isolated from GC tissue samples or cell lines by TRIzol $^{\circledR}$ (Invitrogen) following the manufacturer's protocol. The complementary DNAs (cDNA) were synthesized using the First Strand cDNA Synthesis kit (Roche, Basel, Switzerland), and then performed quantitative PCR analysis by Applied Biosystems ${ }^{\mathrm{TM}}$ PowerUp ${ }^{\mathrm{TM}}$ SYBRTM Green Master Mix kit (Thermo Fisher Scientific, Waltham, MA, USA) in the Applied Biosystems QuantStudio $^{\text {TM }} 12$ K Flex Software (Thermo Fisher Scientific). The primer sequences were as follows: BANCR forward, 5'-ACAGGACTCCATGGCAAACG-3' and BANCR reverse, 5'-ATGAAGAAAGCCTGGTG-3'; GAPDH forward, 5'-ACCACAGTCCATGCCATCAC-3' 
and 5'-TCCACCACCCTGTTGCTGTA-3'. GAPDH acted as an internal standard. The BANCR relative quantitative expression was calculated by the $2^{-\Delta \Delta \mathrm{Ct}}$ methods.

\section{Cell Viability Assays}

We performed cell viability analysis with a Cell Counting Kit-8 (CCK-8, DoJinDo, Tokyo, Japan) according to the manufacturer's protocol. Briefly, 5000 cells/well were seeded in 96-well plates and treated with cisplatin at different concentration gradients for 24 hours. Finally, we measured the absorbance at $450 \mathrm{~nm}$ to determine cell viability. The detailed calculations are as follows: Cell viability $(\%)=[(\mathrm{As}-\mathrm{Ab}) /(\mathrm{Ac}-\mathrm{Ab})] \mathrm{x} 100$; inhibition rate $(\%)=[(\mathrm{Ac}-\mathrm{As}) /(\mathrm{Ac}-\mathrm{Ab})] \mathrm{x} 100 ; \mathrm{As}=\mathrm{OD}$ value of the

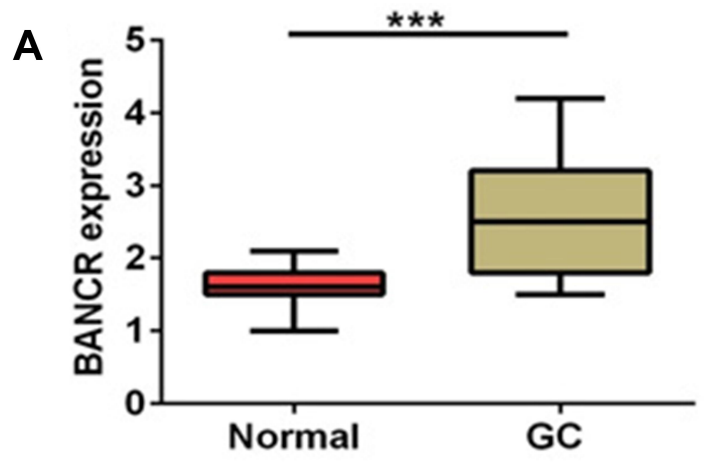

B

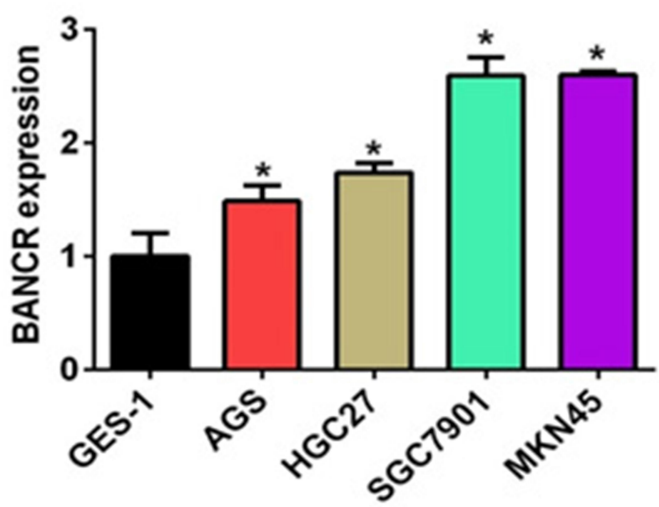

C .흥

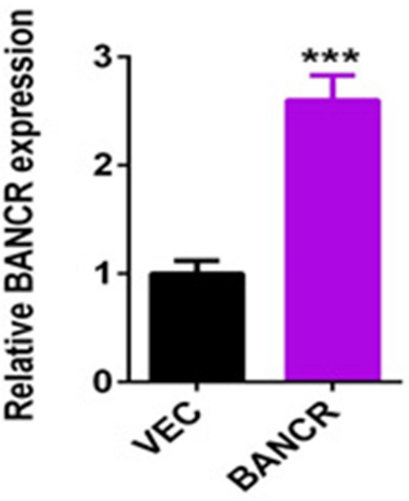

D

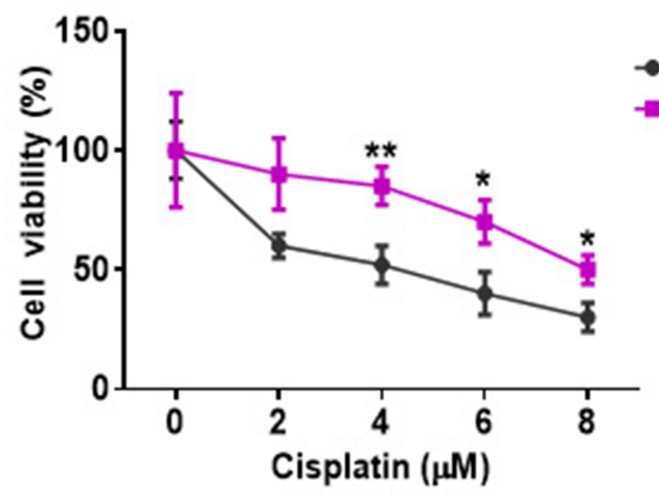

$\mathbf{E}$
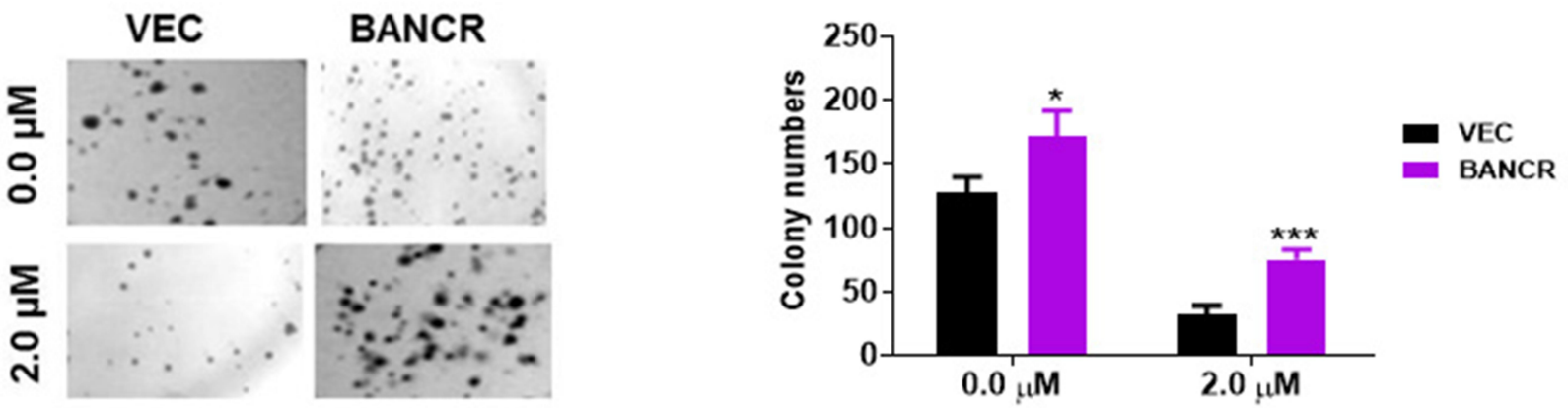

Figure I The expression of BANCR in GC tissues and cancer cell growth. (A) The BANCR level was increased in GC tissues ( $n=35$ ) compared to adjacent normal tissues $(n=35)$. (B) The BANCR level in the human non-malignant gastric epithelial cell line (GES-I) and cancer cell lines (AGS, HGC27, SGC790I and MKN45) were examined by qPCR. Data are represented as the means \pm SD from three independent experiments. (C) The level of BANCR was tested in BANCR-overexpression AGS cells by qPCR analysis. (D) BANCR overexpression increased the cell viability of AGS cells treated with cisplatin. Pictures were taken in light microscope. Magnification is 40X. (E) A clonogenic survival assay was performed using BANCR-overexpression AGS cells treated with cisplatin. Data are represented as the means \pm SD from three independent experiments. ${ }^{*} \mathrm{P}<0.05, * * \mathrm{P}<0.01$, and $* * * \mathrm{P}<0.001$ were considered as significant difference.

Abbreviations: GC, gastric cancer; qPCR, quantity polymerase chain reaction; VEC, only lentivirus vector infected AGS cells; BANCR, AGS cells with BANCR overexpression in lentivirus vector. 
experimental wells; $\mathrm{Ab}=\mathrm{OD}$ value of blank wells; $\mathrm{Ac}=$ OD value of control wells.

\section{Colony Formation Assays}

We seeded GC cell lines into 6-well plates at 500/well density and treated them with cisplatin for 12 days. Fresh media were added into the plates every 3 days. After 12 days, we fixed colonies with $4 \%$ paraformaldehyde and then stained the colonies with $1 \%$ crystal violet. Finally, we photographed and counted the cell colonies.

\section{Western Blot}

The total protein from cultured cells and tumors were obtained with Laemmli sample buffer (Bio-Rad, Hercules, CA, USA), separated by $10 \%$ polyacrylamide gel electrophoresis, and transferred onto polyvinylidene fluoride membranes (Millipore, MA, USA). The protein membrane was incubated overnight at $4^{\circ} \mathrm{C}$ with primary antibodies (anti-pERK1/2, anti-total ERK1/2, anti-pMEK1 /2, anti-MEK1/2, anti-pMER1/2, anti-pSTAT3, antiSTAT3, anti-pAKT, anti-AKT, and anti-GAPDH antibody, Abcam, Cambridge, MA, USA). We used the enhanced chemiluminescence (Thermo Scientific, Waltham, MA) to detect protein bands and semi-quantified the band density with an ImageJ analysis software (National Institutes of Health).

\section{Xenograft Mouse Model}

The lentivirus particles carrying BANCR siRNA-1 sequences (si BANCR-1) were purchased from GenePharma Co. Ltd. We obtained the MKN45 cells with BANCR stably knock-down by infection with si BANCR-1 lentivirus. Then, we subcutaneously injected $5 \times 10^{6}$ MKN45 cells with BANCR stably knock-down or control cells into the back flank of female nude mice (6-8 week-old). Cisplatin $(40 \mu \mathrm{M} / \mathrm{kg})$ was administered intraperitoneally at days 5, 8, 12 and 15 . Tumor size was measured at the indicated days by using a caliper. After 19 days, we isolated the xenograft tumor from mice, photographed the tumor and measured its weight.

\section{Statistical Analysis}

All data were presented as the mean \pm standard deviation (SD). We performed statistical analysis by one tail Student's $t$-test. A P-value less than $0.05,0.01$ or 0.001 was considered significant differences.

\section{Results}

BANCR Expression Was Increased in GC Patients and Cell Lines Compared with Normal Cells

To evaluate BANCR expression in GC and normal cells, we utilized qPCR method for their relative levels. The results revealed that the expression of BANCR in 35 primary GC samples was much higher than corresponding normal tissues. Data is shown in Figure 1A, $(\mathrm{P}<0.001)$. Furthermore, the BANCR level was positively related to tumor node metastasis (TNM) stages $(\mathrm{P}=0.032)$ and lymph node metastasis $(\mathrm{P}=0.045)$ (Table 1). Moreover, it is also shown that BANCR expression was higher in GC cell lines (AGS, HGC27, SGC7901 and MKN45) than normal cells (GES-1) (Figure 1B, $\mathrm{P}<0.05$ ).

\section{BANCR Overexpression-Induced GC Cell Resistance to Cisplatin}

In addition to surgery, chemotherapy is frequently used to treat gastric cancer patients who are in advanced and metastatic stages. Cisplatin and Taxotere are often combined with the other drugs for chemotherapy. ${ }^{13}$ Unfortunately, therapeutic failure eventually occurred because of chemoresistance.

Table I Association Between BANCR Expression and Clinicopathological Features

\begin{tabular}{|l|l|l|l|l|}
\hline Clinicopathological Features & N & \multicolumn{2}{|l|}{$\begin{array}{l}\text { BANCR } \\
\text { Expression }\end{array}$} & \multirow{2}{*}{ P value } \\
\cline { 2 - 4 } & & Low & High & \\
\hline $\begin{array}{l}\text { Age (years) } \\
\leq 60 \\
>60\end{array}$ & 22 & 13 & 9 & \multirow{2}{*}{0.432} \\
\hline $\begin{array}{l}\text { Gender } \\
\text { Female } \\
\text { Male }\end{array}$ & 13 & 6 & 7 & \\
\hline $\begin{array}{l}\text { Tumor size (cm) } \\
\leq 2\end{array}$ & 16 & 7 & 9 & 0.257 \\
$>2$ & 19 & 10 & 9 & \\
\hline $\begin{array}{l}\text { TNM stage } \\
\text { I + II }\end{array}$ & 15 & 7 & 8 & 0.242 \\
III + IV & 20 & 11 & 9 & \\
\hline $\begin{array}{l}\text { Lymph node metastasis } \\
\text { Positive } \\
\text { Negative }\end{array}$ & 13 & 8 & 3 & $0.032^{*}$ \\
\hline
\end{tabular}

Note: *Statistically significant $(P<0.05)$.

Abbreviations: $\mathrm{N}$, case number; TNM, tumor node metastasis. 
To investigate the possible mechanism of BANCR in affecting the cisplatin resistance of GC cells, BANCR overexpression plasmid was transfected into AGS cells. As shown in Figure 1C, BANCR overexpression plasmid could greatly increase BANCR expression in AGS cells. Then, BANCRoverexpression AGS cells were treated with increasing concentrations of cisplatin ( 0 to $8 \mu \mathrm{M})$ for 24 hours. Cisplatin treatment reduced cell viability of AGS cells transfected with or without BANCR overexpression plasmid in a dosedependent manner. However, BANCR-overexpression AGS cells showed higher cell viability (Figure 1D, purple line) compared with control cells (Figure 1D, black line). Moreover, the colony formation assay also showed that BANCR overexpression increased the number of colonies and protected cells from cisplatin killing (Figure 1E).

\section{BANCR Knock-Down Reduced GC Cell Resistance to Cisplatin}

To further assess the functions of BANCR in GC cell resistance to cisplatin, we performed BANCR knock-down in MKN45 cells. As shown in Figure 2A, BANCR siRNAs significantly inhibited BANCR expression in MKN45 cells with three different BANCR targeting sites. Cisplatin treatment reduced cell viability of MKN45 cells transfected with or without si-BANCR-1 in a dose-dependent manner. Interestingly, MKN45 cells treated with si-BANCR-1 showed lower cell viability compared with control cells (Figure 2B). Moreover, the colony formation assay also showed that BANCR knockdown decreased the number of colonies (Figure 2C). These results indicated that BANCR promoted the chemoresistance of cisplatin in GC therapy.
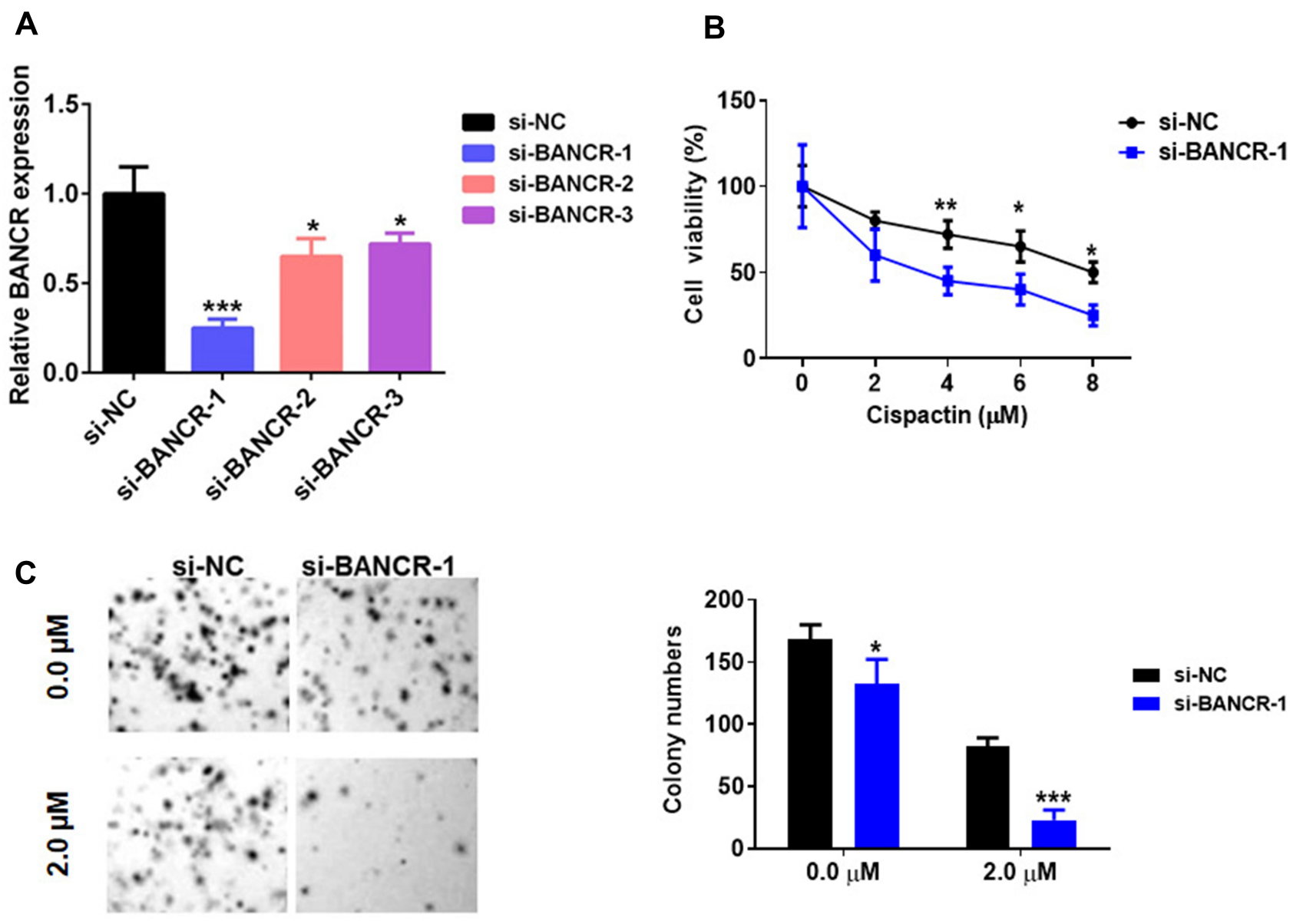

Figure 2 BANCR knockdown inhibited GC cell cisplatin resistance. (A) The level of BANCR was tested in MKN45 cells treated with BANCR siRNAs (BANCR siRNA-I, BANCR siRNA-2 or BANCR siRNA-3) by qPCR analysis. (B) BANCR knockdown decreased the cell viability of MKN45 cells treated with cisplatin. (C) A clonogenic survival assay was performed using MKN45 cotreated with BANCR siRNA-I and cisplatin. Pictures were taken in light microscope. Magnification is 40X. Data are represented as the means \pm SD from three independent experiments. Si-NC, siRNA negative control vector, $* \mathrm{P}<0.05$, $* * \mathrm{P}<0.0 \mathrm{I}$, and $* * * \mathrm{P}<0.00 \mathrm{I}$ were considered as significant difference. 


\section{BANCR Regulated ERKI/2 Signal Pathway in GC Cells}

We next disclosed the molecular pathways involved in the BANCR-mediated resistance to cisplatin in GC cells. Previous studies have shown that BANCR regulates tumor biological behaviors via ERK1/2 signal pathway. ${ }^{14,15}$ Here, we analyzed the ERK1/2 pathway in BANCRoverexpression or BANCR-silenced cells. The results of Western blot showed that BANCR-overexpression markedly up-regulated the phosphorylation level of MEK1/2 and ERK1/2 in AGS cells, but did not affect p-STAT3/ STAT3 and p-AKT/AKT expression (Figure 3A). In contrast, BANCR-knockdown dramatically inhibited the phosphorylation of MEK1/2 and ERK1/2 in MKN45 cells (Figure 3B).

\section{BANCR Overexpression Induced GC Cell Resistance to Cisplatin via ERK Pathway}

We further explored whether BANCR overexpressioninduced cisplatin resistance was ERK1/2 signal pathway dependent. Cell viability analysis showed that BANCRoverexpression enhanced the cisplatin resistance of AGS cells (Figure 4A); while Ly3214996, an inhibitor of ERK signal, treatment abolished the BANCR overexpressionmediated cisplatin resistance (Figure 4A). Moreover, the colony formation assay also confirmed these results (Figure 4B).
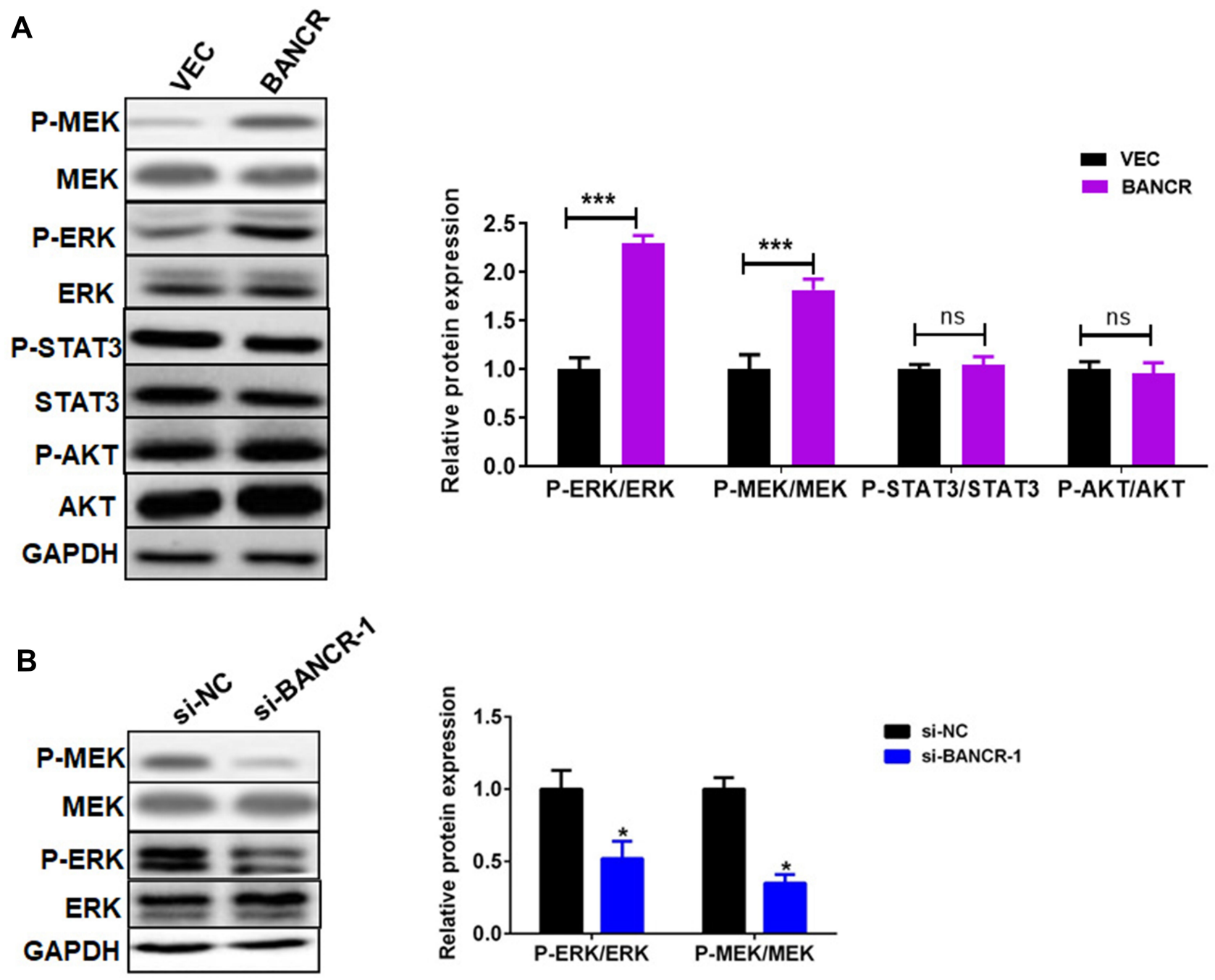

Figure 3 BANCR promoted cisplatin-induced ERKI/2 pathway in GC cells. (A) BANCR overexpression induced the phosphorylation of MEKI/2, ERKI/2, STAT3, and AKT in AGS cells treated with cisplatin. (B) BANCR knockdown reduced the phosphorylation of MEKI/2 and ERKI/2 in MKN45 cells treated with cisplatin. Data are represented as the means $\pm \mathrm{SD}$. $* \mathrm{P}<0.05$ and $* * * \mathrm{P}<0.00$ I were considered as significant difference.

Abbreviations: Vec, only lentivirus vector infected AGS cells; BANCR, AGS cells with BANCR overexpression in lentivirus vector; si-NC, negative control siRNA lentivirus; si-BANCR-I, BANCR-I siRNA lentivirus; ns, no significant difference. 


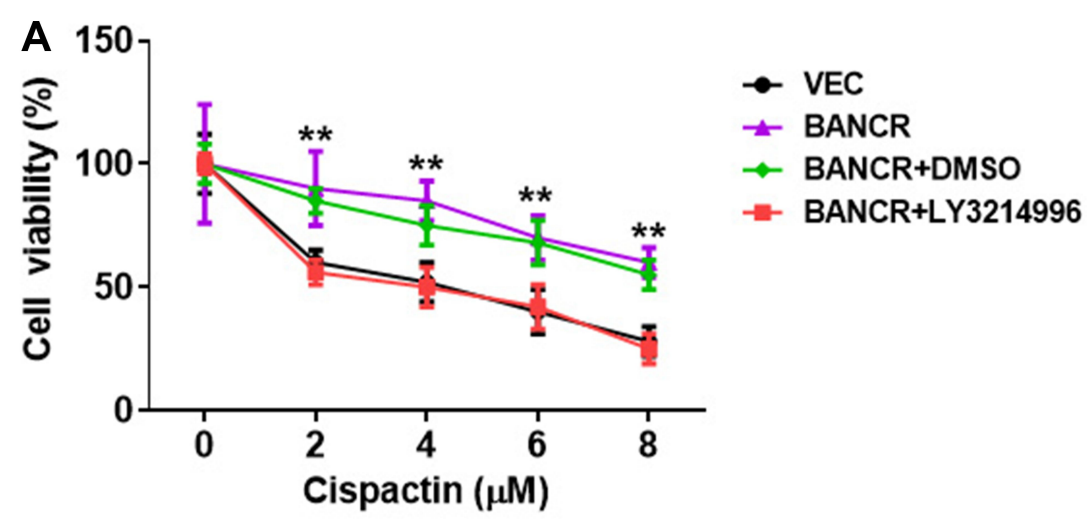

B

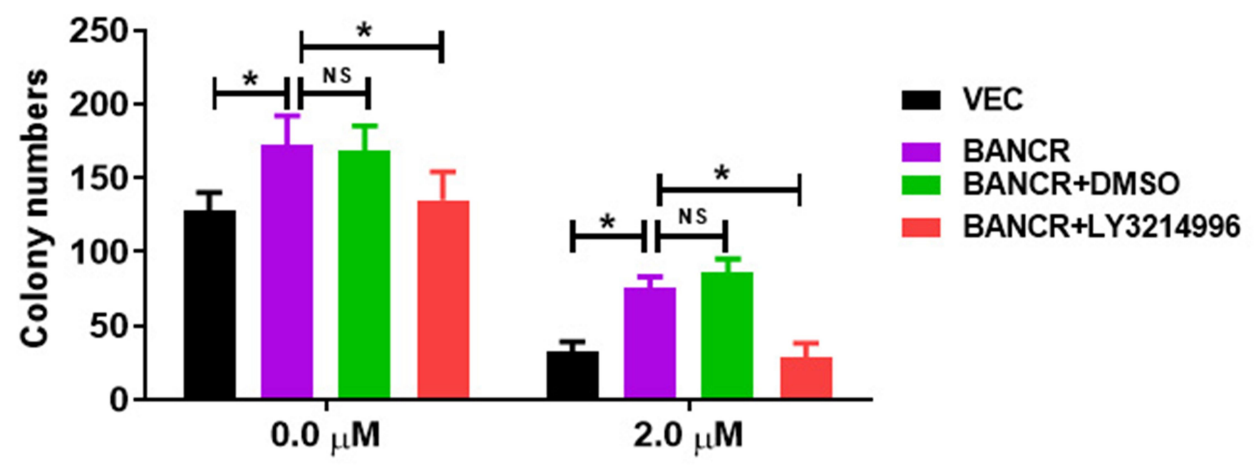

Figure 4 BANCR overexpression induced GC cell resistance to cisplatin via ERK pathway. (A) The increased cell viability of BANCR-overexpression AGS cells treated with cisplatin was abolished by LY3214996. (B) BANCR-overexpression increased the colony numbers of AGS cells treated with cisplatin, while LY32I4996 treatment abolished the effect. Data are represented as the means $\pm S D$. $* P<0.05$, and $* * P<0.01$ were considered as significant difference.

Abbreviations: NS, no significant difference; Vec, only lentivirus vector infected AGS cells; BANCR, AGS cells with BANCR overexpression in lentivirus vector.

\section{BANCR Knockdown Reduced GC Cell Resistance to Cisplatin in vivo}

To investigate the cisplatin resistance effects of BANCR on GC treatment in vivo, we established xenograft models of BANCR knockdown MKN45 tumors in nude mice and treated xenograft models with cisplatin. The images, tumor sizes, and mass showed that tumors from BANCR knockdown MKN45 cells were significantly less resistant to cisplatin than tumors from control MKN45 cells (Figure 5A-C). The expression of BANCR was much lower in BANCR knockdown MKN45 tumors than in control tumors (Figure 5D). Moreover, the phosphorylation levels of MEK1/2 and ERK1/ 2 in BANCR knockdown MKN45 tumors were significantly decreased (Figure 5E).

\section{Discussion}

LncRNAs have been reported to play essential roles in drug resistance in multiple cancers, such as breast cancer, ${ }^{16}$ gastric cancer, ${ }^{17}$ and lung cancer. ${ }^{18}$ For example, lnc-ATB/miR- 200c promoted resistance to trastuzumab and then induced an invasion-metastasis cascade in breast cancer via upregulating ZEB1 and ZNF-217. ${ }^{19}$ Fang et $\mathrm{al}^{20}$ found that knockdown of LEIGC resulted in down-regulated GC cells sensitivity to 5-fluorouracil (5-FU). In lung cancer, the silencing of IncRNA UCA1 promoted gefitinib resistance via targeting FOSL2 signal pathway. ${ }^{21}$ In this study, we found that BANCR promoted GC cell chemoresistance via activating ERK1 pathway both in vitro and in vivo.

Previous studies have shown that aberrant BANCR expression was found in various malignant cancers, including melanoma, endometrial cancer, bladder cancer, and gastric cancer. ${ }^{11,12,22,23}$ The up-regulated BANCR expression was positively associated with clinical stage, tumor depth, lymph node metastasis, and distant metastasis in GC patients. $^{12}$ Here, we also found the expression of BANCR was obviously increased in primary GC samples and cell lines. BANCR was reported to participate in many biological behaviors of a tumor. Shi et $\mathrm{al}^{24}$ showed that 
A

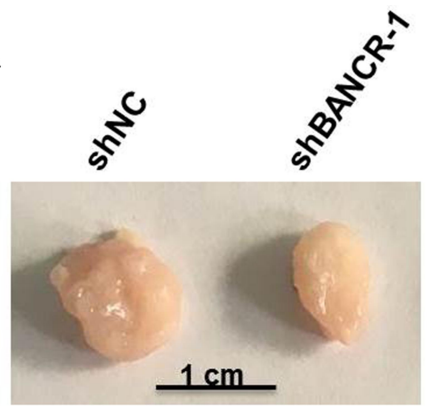

D

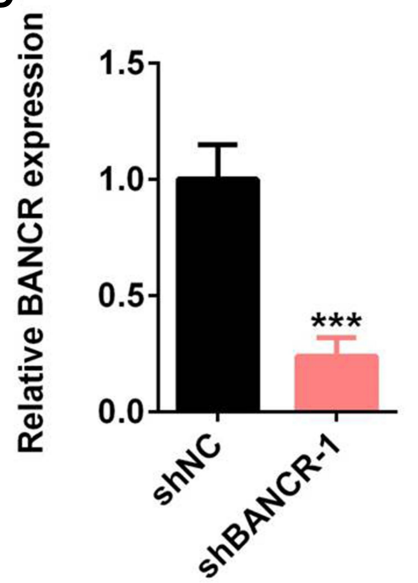

B

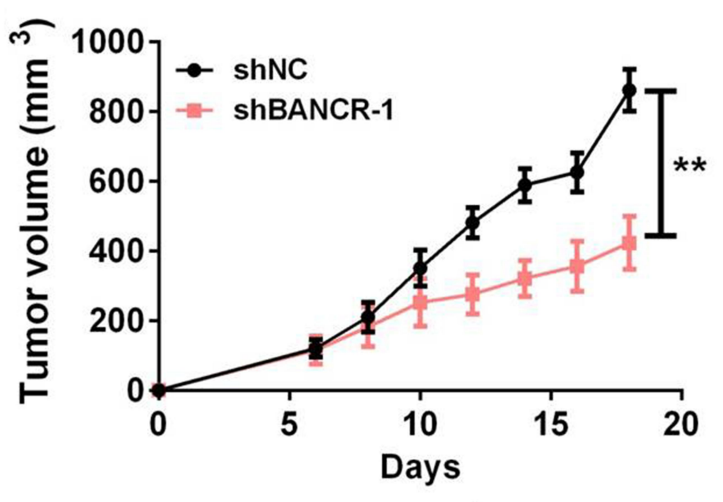

E

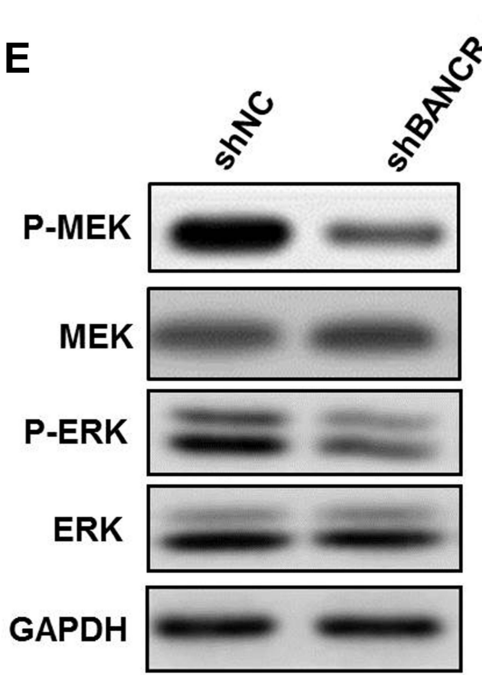

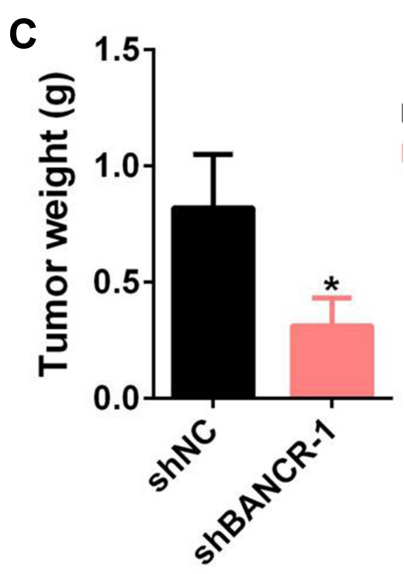

Figure 5 BANCR knockdown reduced GC cell resistance to cisplatin in vivo. (A-C) The tumor images (A), tumor sizes (B), and tumor mass (C) of BANCR knockdown MKN45 tumors treated with cisplatin. (D) The level of BANCR was tested in BANCR knockdown MKN45 tumors by qRT-PCR. (E) The phosphorylation level of MEKI/2 and ERKI/2 in BANCR knockdown MKN45 tumors was tested by Western blot. Data are represented as the means $\pm \mathrm{SD}$. $* \mathrm{P}<0.05$, $* * \mathrm{P}<0.0 \mathrm{I}$, and $* * * \mathrm{P}<0.00 \mathrm{I}$ were considered as significant difference.

Abbreviations: si-NC, negative control siRNA lentivirus infected MKN45 cells; si-BANCR-I, BANCR-I siRNA lentivirus infected MKN45 cells.

BANCR down-regulation contributed to the inhibition of colorectal cancer cell growth through regulating P21 protein. Knockdown of BANCR expression in Huh7 cells using shRNA inhibited the proliferation, apoptosis, migration, and invasion of hepatocellular carcinoma cells. ${ }^{25}$ Moreover, knockdown of BANCR in BGC-823 and MGC-803 cells inhibited cell growth and promoted cell apoptosis. ${ }^{26}$ In this study, BANCR overexpression induced GC cell resistance to cisplatin. In addition, BANCR knockdown reduced GC cell resistance to cisplatin in vivo. Previous study revealed that LncRNA BANCR was involved in chemotherapeutic resistance of colorectal cancer, which mediated sequestering miR-203 molecule. ${ }^{27}$ Our results showed that BANCR played a key role in drug resistance of GC via a different pathway.

ERK1/2 pathway has been reported to be involved in cell growth, malignant transformation and drug resistance. ${ }^{28}$ Overexpression of amphiregulin in ovarian cancer-induced tumor cell stemness and drug resistance through deregulating EGFR-ERK1/2 pathway. ${ }^{29}$ In pancreatic cancer, AGR2 knockdown inhibited the ERK1/2/ AKT axis and further reduced pancreatic cancer cell viability, chemotherapy resistance, migration and invasion. ${ }^{30}$ $\mathrm{Li}$ et $\mathrm{al}^{31}$ found that psoriasin overexpression resulted in the decrease in sensitivity of GC cells to cisplatin by activating the ERK1/2 signal. Therefore, the ERK1/2 pathway plays a pivotal role in tumor drug resistance. Overexpression of BANCR promoted epithelial-mesenchymal transition (EMT), migration, and invasion of BCPAP cells by regulating the Raf/MEK/ERK1/2 signaling. ${ }^{14}$ BANCR regulated cancer stem cell markers in papillary thyroid cancer via the RAF/MEK/ERK1/2 signaling pathway. ${ }^{15}$ Moreover, the role of BANCR in regulating endometrial cancer cell proliferation, migration, and invasion was in an ERK1/2/MAPK signaling pathwaydependent manner. ${ }^{22}$ Therefore, we speculated that 
ERK1/2 signaling may be involved in the BANCRinduced drug resistance of GC cells. Indeed, our results showed that BANCR overexpression markedly upregulated the phosphorylation level of MEK and ERK1/2 in AGS cells. In contrast, BANCR-knockdown inhibited the phosphorylation of ERK1/2 in MKN45 cells. In addition, BANCR knockdown also reduced the phosphorylation level of ERK1/2 in MKN45 tumors in vivo. More importantly, Ly3214996, an inhibitor of ERK signaling, treatment abolished the BANCR overexpression-mediated cisplatin resistance. These data confirmed that the chemotherapeutic resistance of BANCR in cisplatin-treated GC cells was mediated via activating MER/ERK1 pathway.

Previous studies showed that IncRNAs present multiple functions, including post transcriptional regulation and chromatin modification through a miRNA "sponge" ${ }^{, 32} \mathrm{Wu}$ et $\mathrm{al}^{33}$ found that BANCR promoted pancreatic cancer tumorigenesis via modulating miR-195-5p/Wnt/ $\beta$-Catenin signaling pathway. In addition, BANCR induced the proliferation of vascular smooth muscle cells by downregulating miR-34c methylation in atherosclerosis. ${ }^{34}$ Here, we could not exclude the possibility that BANCR may attenuate the killing capacity of cisplatin on GC cells by regulating miRNAs. Further investigation is needed in our future study.

\section{Conclusion}

BANCR was highly expressed in GC tissues and cell lines, and took part in the drug resistance of GC chemotherapy. Both in vitro and in vivo functional experimental analyses showed that BANCR promoted cisplatin resistance of GC cells via the ERK1/2 signaling pathway. This study described a cancer drug resistance property of BANCR and provided a potential therapeutic target of GC.

\section{Abbreviations}

BANCR, BRAF-activated non-coding RNA; CCK-8, cell counting kit-8; cDNA, complementary DNAs; 5-FU, 5-fluorouracil; GAPDH, Glyceraldehyde 3-phosphate dehydrogenase; GC, gastric cancer; NS, no significant difference; LncRNAs, long non-coding RNAs; qPCR, quantity polymerase chain reaction; SD, standard deviation; TNM, tumor node metastasis; VEC, vector.

\section{Data Sharing Statement}

Not applicable.

\section{Ethics Approval and Consent to Participate}

All human gastric cancer sample and animal protocol were approved by the ethical committees of Lianyungang Municipal Oriental Hospital. All patients were given a written inform consent. All animal experimental procedures were performed following guidelines and regulations of the Ethical Committee of Lianyungang Municipal Oriental Hospital.

\section{Consent for Publication}

Not applicable

\section{Author Contributions}

All authors made a significant contribution to the work reported, whether that is in the conception, study design, execution, acquisition of data, analysis and interpretation, or in all these areas; took part in drafting, revising or critically reviewing the article; gave final approval of the version to be published; have agreed on the journal to which the article has been submitted; and agree to be accountable for all aspects of the work.

\section{Disclosure}

All authors declare that there is no conflict of interest.

\section{References}

1. Carcas LP. Gastric cancer review. J Carcinog. 2014;13(1):14. doi:10.4103/1477-3163.146506

2. YiRen H, YingCong Y, Sunwu Y, et al. Long noncoding RNA MALAT1 regulates autophagy associated chemoresistance via miR-23b-3p sequestration in gastric cancer. Mol Cancer. 2017;16 (1):174. doi:10.1186/s12943-017-0743-3

3. Goere D, Gras-Chaput N, Auperin A, et al. Treatment of gastric peritoneal carcinomatosis by combining complete surgical resection of lesions and intraperitoneal immunotherapy using catumaxomab. BMC Cancer. 2014;14(1):148. doi:10.1186/1471-2407-14-148

4. Kalmar A, Nagy ZB, Galamb O, et al. Genome-wide expression profiling in colorectal cancer focusing on lncRNAs in the adenoma-carcinoma transition. BMC Cancer. 2019;19(1):1059. doi:10.1186/s12885-019-6180-5

5. Yang $\mathrm{H}$, Jiang $\mathrm{Z}$, Wang $\mathrm{S}$, et al. Long non-coding small nucleolar RNA host genes in digestive cancers. Cancer Med. 2019;8(18):7693-7704. doi:10.1002/cam 4.2622

6. Barth DA, Slaby O, Klec C, et al. Current concepts of non-coding RNAs in the pathogenesis of non-clear cell renal cell carcinoma. Cancers (Basel). 2019;11(10):1580. doi:10.3390/cancers 11101580

7. Bourguignon LYW. Matrix hyaluronan-CD44 interaction activates microRNA and LncRNA signaling associated with chemoresistance, invasion, and tumor progression. Front Oncol. 2019;9:492. doi: $10.3389 /$ fonc. 2019.00492

8. Zebisch A, Hatzl S, Pichler M, et al. Therapeutic resistance in acute myeloid leukemia: the role of non-coding RNAs. Int J Mol Sci. 2016;17(12):12. doi:10.3390/ijms17122080 
9. Li Y, Ye Y, Feng B, Qi Y. Long noncoding RNA lncARSR promotes doxorubicin resistance in hepatocellular carcinoma via modulating PTEN-PI3K/Akt pathway. J Cell Biochem. 2017;118(12):4498-4507. doi:10.1002/jcb.26107

10. Wu X, Zheng Y, Han B, Dong X. Long noncoding RNA BLACAT1 modulates $\mathrm{ABCB} 1$ to promote oxaliplatin resistance of gastric cancer via sponging miR-361. Biomed Pharmacother. 2018;99:832-838. doi:10.1016/j.biopha.2018.01.130

11. Flockhart RJ, Webster DE, Qu K, et al. BRAFV600E remodels the melanocyte transcriptome and induces BANCR to regulate melanoma cell migration. Genome Res. 2012;22(6):1006-1014. doi:10.1101/ gr.140061.112

12. Li L, Zhang L, Zhang Y, Zhou F. Increased expression of LncRNA BANCR is associated with clinical progression and poor prognosis in gastric cancer. Biomed Pharmacother. 2015;72:109-112. doi:10.1016/j.biopha.2015.04.007

13. Wagner AD, Syn NL, Moehler M, et al. Chemotherapy for advanced gastric cancer. Cochrane Database Syst Rev. 2017;8:CD004064. doi:10.1002/14651858.CD004064.pub4

14. Wang Y, Gu J, Lin X, et al. IncRNA BANCR promotes EMT in PTC via the Raf/MEK/ERK signaling pathway. Oncol Lett. 2018;15 (4):5865-5870. doi:10.3892/ol.2018.8017

15. Wang $\mathrm{Y}, \mathrm{Lin} \mathrm{X}, \mathrm{Fu} \mathrm{X}$, et al. Long non-coding RNA BANCR regulates cancer stem cell markers in papillary thyroid cancer via the RAF/MEK/ERK signaling pathway. Oncol Rep. 2018;40(2):859-866. doi:10.3892/or.2018.6502

16. Chen Z, Pan T, Jiang D, et al. The lncRNA-GAS5/miR-221-3p/ DKK2 axis modulates abcb1-mediated adriamycin resistance of breast cancer via the Wnt/beta-catenin signaling pathway. Mol Ther Nucleic Acids. 2020;19:1434-1448. doi:10.1016/j.omtn.2020.01.030

17. Wei L, Sun J, Zhang N, et al. Noncoding RNAs in gastric cancer: implications for drug resistance. Mol Cancer. 2020;19(1):62. doi:10.1186/s12943-020-01185-7

18. Mondal P, Natesh J, Kamal MA, Meeran SM. Non-coding RNAs in lung cancer chemoresistance. Curr Drug Metab. 2020;20 (13):1023-1032. doi:10.2174/1389200221666200106105201

19. Shi S, Wang L, Yu B, et al. LncRNA-ATB promotes trastuzumab resistance and invasion-metastasis cascade in breast cancer. Oncotarget. 2015;6(13):11652-11663. doi:10.18632/oncotarget.3457

20. Fang X, Pan H, Leng R, Ye D. Long noncoding RNAs: novel insights into gastric cancer. Cancer Lett. 2015;356(2):357-366. doi:10.1016/j. canlet.2014.11.005

21. Chen X, Wang Z, Tong F, et al. IncRNA UCA1 promotes gefitinib resistance as a ceRNA to target FOSL2 by sponging miR-143 in non-small cell lung cancer. Mol Ther Nucleic Acids. 2020;19:643-653. doi:10.1016/j.omtn.2019.10.047

22. Wang D, Wang D, Wang N, et al. Long non-coding RNA BANCR promotes endometrial cancer cell proliferation and invasion by regulating MMP2 and MMP1 via ERK/MAPK signaling pathway. Cell Physiol Biochem. 2016;40(3-4):644-656.
23. He A, Liu Y, Chen Z, et al. Over-expression of long noncoding RNA BANCR inhibits malignant phenotypes of human bladder cancer. $J$ Exp Clin Cancer Res. 2016;35(1):125. doi:10.1186/s13046-0160397-9

24. Shi Y, Liu Y, Wang J, et al. Downregulated long noncoding RNA BANCR promotes the proliferation of colorectal cancer cells via downregulation of p21 expression. PLoS One. 2015;10(4): e0122679. doi:10.1371/journal.pone.0122679

25. Li J, Wang J, Zhou W, et al. Downregulation of BRAF-activated non-coding RNA suppresses the proliferation, migration and invasion, and induces apoptosis of hepatocellular carcinoma cells. Oncol Lett. 2017;14(4):4751-4757. doi:10.3892/o1.2017.6770

26. Zhang ZX, Liu ZQ, Jiang B, et al. BRAF activated non-coding RNA (BANCR) promoting gastric cancer cells proliferation via regulation of NF-kappaB1. Biochem Biophys Res Commun. 2015;465 (2):225-231. doi:10.1016/j.bbrc.2015.07.158

27. Ma S, Yang D, Liu Y, et al. LncRNA BANCR promotes tumorigenesis and enhances adriamycin resistance in colorectal cancer. Aging (Albany NY). 2018;10(8):2062-2078. doi:10.18632/aging.101530

28. McCubrey JA, Steelman LS, Chappell WH, et al. Roles of the Raf/ MEK/ERK pathway in cell growth, malignant transformation and drug resistance. Biochem Biophys Acta. 2007;1773(8):1263-1284.

29. Tung S-L, Huang W-C, Hsu F-C, et al. miRNA-34c-5p inhibits amphiregulin-induced ovarian cancer stemness and drug resistance via downregulation of the AREG-EGFR-ERK pathway. Oncogenesis. 2017;6(5):e326. doi:10.1038/oncsis.2017.25

30. Liu QG, Li YJ, Yao L. Knockdown of AGR2 induces cell apoptosis and reduces chemotherapy resistance of pancreatic cancer cells with the involvement of ERK/AKT axis. Pancreatology. 2018;18 (6):678-688. doi:10.1016/j.pan.2018.07.003

31. Li L, Cui Y, Ye L, et al. Psoriasin overexpression confers drug resistance to cisplatin by activating ERK in gastric cancer. Int J Oncol. 2018;53(3):1171-1182. doi:10.3892/ijo.2018.4455

32. Kapusta A, Feschotte C. Volatile evolution of long noncoding RNA repertoires: mechanisms and biological implications. Trends Genet. 2014;30(10):439-452. doi:10.1016/j.tig.2014.08.004

33. Wu X, Xia T, Cao M, et al. LncRNA BANCR promotes pancreatic cancer tumorigenesis via modulating MiR-195-5p/Wnt/beta-catenin signaling pathway. Technol Cancer Res Treat. 2019;18:1533033819887962. doi:10.1177/1533033819887962

34. Jiang X, Liu Z, Qi X. LncRNA BANCR induced vascular smooth muscle cell proliferation by downregulating miR-34c methylation in atherosclerosis. J Thromb Thrombolysis. 2020. doi:10.1007/s11239020-02314-1

\section{Publish your work in this journal}

Cancer Management and Research is an international, peer-reviewed open access journal focusing on cancer research and the optimal use of preventative and integrated treatment interventions to achieve improved outcomes, enhanced survival and quality of life for the cancer patient.
The manuscript management system is completely online and includes a very quick and fair peer-review system, which is all easy to use. Visit http://www.dovepress.com/testimonials.php to read real quotes from published authors. 